\title{
TRPV4 is functionally expressed in oligodendrocyte precursor cells and increases their proliferation
}

\section{AUTHOR(S):}

Ohashi, Kana; Deyashiki, Ayane; Miyake, Takahito; Nagayasu, Kazuki; Shibasaki, Koji; Shirakawa, Hisashi; Kaneko, Shuji

\section{CITATION:}

Ohashi, Kana ... [et al]. TRPV4 is functionally expressed in oligodendrocyte precursor cells and increases their proliferation. Pflügers Archiv - European Journal of Physiology 2018, 470(5): 705-716

\section{ISSUE DATE:}

2018-05

URL:

http://hdl.handle.net/2433/241626

\section{RIGHT:}

This is a post-peer-review, pre-copyedit version of an article published in Pflügers Archiv - European Journal of Physiology. The final authenticated version is available online at: http://dx.doi.org/10.1007/s00424-018-2130-3.; この論 文は出版社版でありません。引用の際には出版社版をご確認ご利用ください。;This is not the published version. Please cite only the published version. 


\section{TRPV4 is functionally expressed in oligodendrocyte precursor cells and increases their proliferation}

Kana Ohashi $^{1 *}$, Ayane Deyashiki ${ }^{1 *}$, Takahito Miyake ${ }^{1}$, Kazuki Nagayasu$^{1}$, Koji Shibasaki $^{2}$, Hisashi Shirakawa $^{1 \#}$, and Shuji Kaneko ${ }^{1}$

${ }^{1}$ Department of Molecular Pharmacology, Graduate School of Pharmaceutical Sciences, Kyoto University, Kyoto 606-8501, Japan

${ }^{2}$ Department of Molecular and Cellular Neurology, Graduate School of Medicine, Gunma University, Maebashi 371-8511, Japan

*These authors contributed equally to this work.

\#Correspondence to:

Hisashi Shirakawa, PhD, 46-29 Yoshida-shimoadachi-cho, Sakyo-ku, Kyoto, 606-8501, Japan; Tel: +81-75-753-4549; Fax: +81-75-753-4548; E-mail: shirakaw@pharm.kyoto-u.ac.jp

Key words: TRPV4 channel, Oligodendrocyte precursor cells, $\mathrm{Ca}^{2+}$ imaging, Protein kinase C, Cell proliferation

Acknowledgments: We appreciate Dr. H. Takebayashi (Niigata Univ.) for the generous gift of PDGFR $\alpha$, plp and gfap ISH plasmids, and Dr. K. Arai (Harvard Univ.) for his excellent advice on primary culture of oligodendrocyte precursor cells. This work was supported by MEXT/JSPS KAKENHI Grant Numbers 17K19486 (to H.S.), 24390016 (to S.K.), JP15H05934 <Thermal Biology> (to K.S.), JP15H03000 (to K.S.), and also supported by the Takeda Science Foundation and the Mochida Memorial Foundation for Medical and Pharmaceutical Research (to H.S.). 


\section{Abstract}

Oligodendrocytes, which differentiate from oligodendrocyte precursor cells (OPCs), ensheath axons with myelin, play an essential role in rapid conduction of action potentials, and metabolically support neurons. Elucidation of the mechanisms underlying the proliferation, migration, differentiation, and survival of OPCs is considered indispensable for determining the causes of central nervous system diseases. However, the relationship between these functions of OPCs and their intracellular $\mathrm{Ca}^{2+}$ signaling has not been fully elucidated. Here, we investigated the function of transient receptor potential vanilloid 4 (TRPV4), a $\mathrm{Ca}^{2+}$-permeable channel that responds to hypo-osmolarity, mild temperature, mechanical stimulation, and endogenous arachidonic acid metabolites, in OPCs. Trpv4 mRNA was detected in OPCs in vivo and in primary cultured rat OPCs. In $\mathrm{Ca}^{2+}$ imaging experiments, treatment with the selective TRPV4 agonist GSK1016790A induced sustained elevation of the intracellular $\mathrm{Ca}^{2+}$ concentration in OPCs in a concentration-dependent manner, which was almost completely suppressed by co-treatment with the selective TRPV4 antagonist HC067047. Stimulation of TRPV4 by GSK1016790A augmented OPC proliferation, which was abolished by co-treatment with HC067047, the intracellular $\mathrm{Ca}^{2+}$ chelator BAPTA-AM, and the protein kinase C inhibitor bisindolylmaleimide II. By contrast, GSK1016790A did not significantly affect the migration or differentiation of OPCs. Taken together, these results suggest that TRPV4 is functionally expressed in OPCs and increases the proliferation of these cells without affecting their ability to differentiate into oligodendrocytes. 


\section{INTRODUCTION}

Oligodendrocytes are a type of glial cell and ensheath axons with myelin. Consequently, they not only allow rapid conduction of electrical impulses [45], but also metabolically support neurons [23]. Oligodendrocyte precursor cells (OPCs) proliferate, migrate, and differentiate into oligodendrocytes. Demyelination and white matter injury are observed in various central nervous system (CNS) diseases, such as multiple sclerosis [15], Alzheimer's disease [14], and psychiatric diseases [31]. Therefore, appropriate regulation of the survival, proliferation, migration, and differentiation of OPCs can effectively improve the pathology of these diseases. Proliferation of OPCs is the first step in remyelination after CNS injury. OPCs die in multiple sclerosis, leading to poor remyelination [9]. Recent reports indicate that OPCs not only differentiate into myelinating oligodendrocytes, but also secrete brain-derived neurotrophic factor (BDNF) and protect neurons against apoptosis [5, 43]. Therefore, increasing the number of OPCs is considered a major strategy to promote remyelination and functional recovery after CNS damage [11, 22].

Although the intracellular signaling that regulates OPC functions remains to be clarified, many studies have investigated molecules that influence these behaviors, such as sonic hedgehog, Wnt proteins, $N$-methyl-D-aspartate (NMDA), platelet-derived growth factor (PDGF), fibroblast growth factor (FGF), neuregulin, and BDNF [3, 13, 24, 25, 32]. Several studies reported that AMPA receptors [10], P2X purine receptors [16], and voltage-dependent $\mathrm{Ca}^{2+}$ channels [6] elevate the intracellular $\mathrm{Ca}^{2+}$ concentration $\left(\left[\mathrm{Ca}^{2+}\right]_{\mathrm{i}}\right)$ in OPCs. Stimulation of these receptors/channels modulates the proliferation [6], morphology [10], differentiation, and myelination [6] of OPCs. Moreover, PDGF and FGF induce $\mathrm{Ca}^{2+}$ responses [33]. However, the detailed $\mathrm{Ca}^{2+}$ signaling mechanisms have not been fully understood.

Transient receptor potential (TRP) channels are $\mathrm{Ca}^{2+}$-permeable, non-selective, cation 
channels with multiple functions. Twenty-eight mammalian TRP genes comprising six families (TRPC, TRPM, TRPV, TRPA, TRPP, and TRPML) have been identified [46]. Some TRP channels have been detected in oligodendrocyte lineage cells. For example, TRPC1 activation is involved in store-operated $\mathrm{Ca}^{2+}$ influx in OPCs and increases their proliferation [34]. At the onset of myelination, TRPM3 is expressed in oligodendrocytes and its activation induces $\mathrm{Ca}^{2+}$ responses in these cells [18]. TRPA1 is functionally expressed in mature oligodendrocytes, and an increase in the $\left[\mathrm{Ca}^{2+}\right]_{\mathrm{i}}$ via this proton-gated channel contributes to myelin damage [17]. However, the relationship between the other TRP channels and the functions of oligodendrocyte lineage cells is poorly understood.

This study focused on transient receptor potential vanilloid 4 (TRPV4), which can sense a variety of environmental stimuli such as temperature, osmolarity, mechanical stretch, and arachidonic acid metabolites. We report that TRPV4 is expressed in OPCs in vivo and in primary cultured rat OPCs. Additionally, stimulation of TRPV4 by its selective agonist GSK1016790A increased OPC proliferation via $\mathrm{Ca}^{2+}$ influx and the protein kinase C (PKC) pathway. Our results indicate that TRPV4 increases the proliferation of OPCs without affecting their ability to differentiate into mature oligodendrocytes. 


\section{MATERIALS AND METHODS}

\section{Fluorescence in situ hybridization (FISH) and immunohistochemistry}

Immunohistochemistry and fluorescence in situ hybridization (FISH) were performed as previously described [38, 39]. The Trpv4, PDGFRa, plp and gfap cRNA riboprobes were described previously [26, 27, 38]. Biotin- and digoxigenin-labeled cRNA riboprobes were prepared as previously described [39]. Sagittal sections of 8-week-old mouse brains were hybridized with a mixture of biotin- and digoxigenin-labeled cRNA probes in hybridization buffer. Following washing, digoxigenin and biotin were detected via a two-step method. Biotin was first detected with peroxidase-conjugated streptavidin (PerkinElmer; 1:100 dilution, $1 \mathrm{~h}$ ) and a fluorescein isothiocyanate-tyramide signal amplification (TSA) Plus Kit (PerkinElmer). Then, after incubation with $1.0 \% \mathrm{H}_{2} \mathrm{O}_{2}$ for 30 min to inactivate residual peroxidase, digoxigenin was detected by labeling with a peroxidase-conjugated anti-digoxigenin antibody (Roche Diagnostics; 1:500 dilution, $1 \mathrm{~h}$ ) and an indocarbocyanine-TSA Plus Kit (PerkinElmer). An anti-Iba1 antibody (1:500 dilution, Wako) and Alexa Flour 564-conjugated goat anti-rabbit IgG (1:2000 dilution) were used to detect Iba1 after Trpv4 FISH. Sections were examined using a fluorescence microscope (BX53, Olympus) equipped with a cooled CCD camera (DP80, Olympus).

\section{Primary culture of OPCs}

All experiments were conducted in accordance with the ethical guidelines set down by the Kyoto University Animal Research Committee. Primary OPC cultures were prepared from cerebral cortices of 0-2-day-old Wistar/ST rats (Japan SLC). After isolation of cerebral cortices, cells were incubated with trypsin (2.5 mg/mL; Nacalai Tesque) and DNase I (25 $\mu \mathrm{g} / \mathrm{mL}$; D5025, 
Sigma-Aldrich) for $15 \mathrm{~min}$ at $37^{\circ} \mathrm{C}$. Dissociated cells were plated onto poly-L-ornithine-coated $75 \mathrm{~cm}^{2}$ flasks in Dulbecco's modified Eagle’s medium (D5796, Sigma-Aldrich) containing 10\% heat-inactivated fetal bovine serum and 1\% penicillin/streptomycin mixed solution (Nacalai Tesque) and maintained at $37^{\circ} \mathrm{C}$ in a humidified atmosphere containing $5 \% \mathrm{CO}_{2}$. After $10-15$ days, OPCs were purified from the mixed glial culture via a two-step procedure. First, microglia were removed by shaking at 120-130 rpm for $1 \mathrm{~h}$ using an orbital shaker. After removing the supernatant and adding fresh culture medium, OPCs on a monolayer of astrocytes were detached by shaking at 230-250 rpm for 16-18 h. To remove astrocytes, the supernatant was cultured on non-coated $10 \mathrm{~cm}$ dishes for $1 \mathrm{~h}$ at $37^{\circ} \mathrm{C}$ in $5 \% \mathrm{CO}_{2}$. The final cell suspension was replated onto poly-L-ornithine-coated $10 \mathrm{~mm}$ glass coverslips, $35 \mathrm{~mm}$ dishes, or 48 well plates and maintained in Neurobasal medium (Life Technologies) supplemented with $1 \times$ B27 (Life Technologies), glutamine (2 mM), 1\% penicillin/streptomycin mixed solution, PDGF-AA (10 ng/mL; PeproTech), and basic FGF (10 ng/mL; PeproTech), which was used as proliferation medium. Cells were used for experiments at least 1 day after replating. To analyze OPC differentiation, 2 days after replating, proliferation medium was changed to Neurobasal medium supplemented with $1 \times \mathrm{B} 27$, glutamine $(2 \mathrm{mM}), 1 \%$ penicillin/streptomycin mixed solution, triiodo-L-thyronine (T3; $30 \mathrm{ng} / \mathrm{mL}$; Sigma-Aldrich), and ciliary neurotrophic factor (CNTF; 10 ng/mL; PeproTech), which was used as differentiation medium.

\section{Immunocytochemistry}

OPCs were maintained on $10 \mathrm{~mm}$ glass coverslips for 2 days. To analyze differentiation, cells were maintained for an additional 5 days in differentiation medium. Following fixation in 4\% paraformaldehyde, cells were permeabilized in phosphate-buffered saline (PBS) containing 3\% bovine serum albumin and $0.1 \%$ Triton X-100 for $30 \mathrm{~min}$. To detect platelet-derived growth 
factor receptor $\alpha$ (PDGFR $\alpha$ ) and myelin basic protein (MBP), cells were incubated with a rabbit polyclonal anti-PDGFR $\alpha$ antibody (1:1000; Santa Cruz) and a rat anti-MBP antibody (1:375; Millipore) for $3 \mathrm{~h}$ at room temperature, and then with Alexa Fluor 594-labeled donkey anti-rabbit IgG (1:500; Invitrogen) and Alexa Fluor 488-labeled donkey anti-rat IgG (1:500; Invitrogen) overnight at $4^{\circ} \mathrm{C}$. After staining nuclei with DAPI Fluoromount-G (Southern Biotechnology Associates), fluorescence images were acquired using a FluoView FV10i confocal microscope (Olympus).

\section{Reverse transcription (RT)-PCR}

Total RNA was extracted from OPCs cultured in a $35 \mathrm{~mm}$ dish using a Nucleo Spin RNA kit (TaKaRa Bio). First-strand cDNA was prepared from total RNA using a ReverTra Ace qPCR RT Kit (Toyobo). Rat Trpv4 cDNA was amplified by PCR using Blend Taq (Toyobo) and the following primer pair: 5'-GGTTGTGTCTGCGGCGCTCT-3' and 5'-GTGGTGGCCCACTGCAGCTT-3'. The PCR conditions were as follows: $94^{\circ} \mathrm{C}$ for $2 \mathrm{~min}$, followed by 30 cycles of $94^{\circ} \mathrm{C}$ for $30 \mathrm{~s}, 58^{\circ} \mathrm{C}$ for $30 \mathrm{~s}$, and $72^{\circ} \mathrm{C}$ for $1 \mathrm{~min}$. Amplified PCR products were electrophoresed in an agarose gel, stained with $0.1 \mu \mathrm{g} / \mathrm{mL}$ ethidium bromide, and visualized under ultraviolet light. Images were captured with Quantity One software (Bio-Rad).

\section{Western Blotting}

Western blotting was performed as described previously [42], with slight modification. After 2 days in enriched culture, cells were lysed in RIPA buffer (Nacalai Tesque). Proteins were diluted in NuPAGE ${ }^{\circledR} \quad 4 \mathrm{x}$ LDS sample buffer (Life Technologies), loaded onto a $10 \%$ SDS-polyacrylamide gel, and blotted onto Immobilon-P PVDF transfer membranes (Millipore). After a blocking step using Blocking One (Nacalai tesque), the membranes were incubated 
overnight at $4{ }^{\circ} \mathrm{C}$ with anti-TRPV4 antibody (ab39260, Abcam), diluted 1:250 in Tris-buffered saline containing $0.1 \%$ Tween-20 and $10 \%$ Blocking One. After washing, membranes were incubated with peroxidase-conjugated donkey anti-rabbit IgG (1:5000 dilution; GE Healthcare) for $1 \mathrm{~h}$ at RT. Specific bands were detected by Immobilon Western Chemiluminescent HRP substrate (Millipore) and visualized using the ChemiImager 4400 imaging system (Alpha Innotech).

\section{Fluorometric $\mathrm{Ca}^{2+}$ imaging}

$\mathrm{Ca}^{2+}$ imaging was conducted as previously reported [42] with slight modification. OPCs on coverslips were loaded for 30-40 min with $5 \mu \mathrm{M}$ fura-2 acetoxymethyl ester (Fura-2 AM; Dojindo) prepared in Krebs-Ringer buffer (140 mM NaCl, $5 \mathrm{mM} \mathrm{KCl,} 1 \mathrm{mM} \mathrm{MgCl}$, $2 \mathrm{mM}$ $\mathrm{CaCl}_{2}, 10 \mathrm{mM}$ glucose, and $10 \mathrm{mM}$ HEPES, adjusted to $\mathrm{pH}$ 7.4) containing $0.005 \%$ Cremophor EL (Sigma-Aldrich). Fluorescence images were captured at room temperature every $2 \mathrm{~s}$ with the AQUACOSMOS/ORCA-AG imaging system (Hamamatsu Photonics) using alternating excitation wavelengths of 340 and $380 \mathrm{~nm}$ and an emission wavelength of $510 \mathrm{~nm}$. The ratio of the fluorescence intensity obtained with excitation/emission wavelengths of $340 \mathrm{~nm} / 510 \mathrm{~nm}$ (F340) to that obtained with excitation/emission wavelengths of $380 \mathrm{~nm} / 510 \mathrm{~nm}$ (F380), so-called F340/F380, was calculated to quantify the $\left[\mathrm{Ca}^{2+}\right]_{\mathrm{i}}$.

\section{Whole-cell patch-clamp recording}

Whole-cell currents were recorded at room temperature with a pipette made from a $1.5 \mathrm{~mm}$ outer-diameter glass capillary with a filament (Narishige) and pulled using a P-87 micropipette puller (Sutter) as previously described [29] with slight modification. Access resistance ranged from 2 to $5 \mathrm{M} \Omega$ when the pipette was filled with the intracellular solution described below. The 
bath solution contained $150 \mathrm{mM} \mathrm{NaCl}, 1 \mathrm{mM} \mathrm{MgCl}$, $1.5 \mathrm{mM} \mathrm{CaCl}$, $10 \mathrm{mM}$ HEPES, and 10 mM D-glucose (adjusted to pH 7.4 with $\mathrm{NaOH}$ ). The pipette solution contained $150 \mathrm{mM} \mathrm{CsCl}$, $10 \mathrm{mM}$ BAPTA $1 \mathrm{mM} \mathrm{MgCl}_{2}$, and $10 \mathrm{mM}$ HEPES (adjusted to $\mathrm{pH} 7.2$ with $\mathrm{CsOH}$ ). Current-voltage relationships were measured using voltage ramps ( -100 to $+100 \mathrm{mV}$ over 200 ms, $0.2 \mathrm{~Hz}$ ). The holding potential was set to $0 \mathrm{mV}$. Access resistance was compensated by $70 \%$. Patch-clamp recordings were performed using an EPC-10 patch-clamp amplifier (HEKA Instruments) and PATCHMASTER software (HEKA). Data were filtered at $2.9 \mathrm{kHz}$ for analysis.

\section{Scratch-wound assay}

When OPCs in 35 mm dishes were $70-80 \%$ confluent, a wound was created using a cell scraper (BM Bio). Cultures were washed twice with PBS, medium containing GSK1016790A or vehicle (dimethyl sulfoxide) was added, and cells were allowed to migrate for 6-48 h. Phase contrast images were acquired using a DIAPHOT 300 inverted phase contrast microscope (Nikon) and a DS-U1 DIGITAL SIGHT camera system (Nikon). The number of cells that migrated into the scratched area was counted.

\section{MTT assay}

Cell proliferation/viability was assessed by the MTT (3-(4,5-dimethyl-2-thiazolyl)-2,5-diphenyltetrazolium bromide) assay according to the manufacturer's protocol (Nacalai Tesque). Twenty-four to $72 \mathrm{~h}$ after the drug treatment, cells were treated with MTT $(0.025 \mathrm{mg} / \mathrm{mL})$ for $3 \mathrm{~h}$, crystals were solubilized in dimethyl sulfoxide, and absorbance at $570 \mathrm{~nm}$ was measured. Cell viability was expressed as a ratio in comparison with non-treated control cells. 


\section{Incorporation of bromodeoxyuridine (BrdU) and fluorescence staining}

Two days after replating onto $10 \mathrm{~mm}$ glass coverslips, OPCs were treated with drugs for $24 \mathrm{~h}$. Thereafter, OPCs were treated with bromodeoxyuridine (BrdU; $1 \mu \mathrm{M}$; Sigma-Aldrich) for $3 \mathrm{~h}$, fixed in $4 \%$ paraformaldehyde, and soaked in $1 \mathrm{~N} \mathrm{HCl}$ for $20 \mathrm{~min}$ at $37^{\circ} \mathrm{C}$ to denature nuclear DNA. Then, cells were incubated with a mouse monoclonal anti-BrdU antibody (1:500; Roche) for $3 \mathrm{~h}$ at room temperature followed by Alexa Fluor 488-labeled goat anti-mouse IgG (1:500; Invitrogen) overnight at $4^{\circ} \mathrm{C}$. After staining nuclei with DAPI Fluoromount-G, fluorescence images were acquired using a FluoView FV10i confocal microscope (Olympus). The percentage of $\mathrm{BrdU}^{+}$cells was estimated based on the total number of cells.

\section{Quantitative RT-PCR}

After reverse transcription of total mRNA into cDNA using a ReverTra Ace qPCR RT Kit, real-time quantitative PCR was performed using the StepOne Real-Time PCR System (Life Technologies) and THUNDERBIRD SYBR qPCR Mix (Toyobo). The primer sets were as follows: 5'-CGGTCCAAGAATTTCACCTC-3' and 5'-ACCGCGGTTCTATTTTGTTG-3' for rat 18S ribosomal RNA (rRNA), 5'-GATAGCTTCATGAGCCGACACCCA-3' and 5'-CATTGGCACGTACTGTGTGGTGTCA-3' for rat Pdgfra, and 5'-CTCTGGCAAGGACTCACACA-3' and 5'-TGTCTCTTCCTCCCCAGCTA-3' for rat Mbp. The PCR conditions were as follows: $95^{\circ} \mathrm{C}$ for $10 \mathrm{~min}$, followed by 45 cycles of $95^{\circ} \mathrm{C}$ for $15 \mathrm{~s}$ and $60^{\circ} \mathrm{C}$ for $60 \mathrm{~s}$. Expression of each gene was normalized against that of $18 \mathrm{~S}$ rRNA, which was measured in parallel in each sample.

\section{Statistical analysis}


All data were expressed as means \pm SEM. Statistical analyses were performed by Student's t-test or one-way ANOVA followed by Tukey's test using GraphPad Prism 5. In all cases, $P<0.05$ was considered statistically significant. 


\section{RESULTS}

\section{OPCs express TRPV4 in vivo}

We previously reported that astrocytes and microglial cells express TRPV4, in addition to neurons [21, 38, 40], and we are keen to understand whether OPCs express TRPV4. To address this possibility, we examined Trpv4 and PDGFR $\alpha$ (an OPC marker) mRNA in cerebral cortex (gray matter) and corpus callosum (white matter) in P4 or adult rats by FISH (Fig. 1A), and detected Trpv4 in most PDGFR $\alpha^{+}$OPCs ( $\sim 80 \%$, arrowheads); however, some OPCs ( $20 \%$, arrows) did not exhibit clear Trpv4 FISH signals at P4. The numbers of Trpv4/PDGFR $\alpha$ double-positive cells were not different between cerebral cortex and corpus callosum (Fig. 1A). In adult, the numbers were significantly reduced in the cerebral cortex, and we observed many Trpv4/PDGFR $\alpha$ double-positive cells in the corpus callosum (Fig. 1A), indicating that OPCs express TRPV4, as expected. We also performed double FISH of Trpv4 and Plp. The Plp ${ }^{+}$ mature oligodendrocytes displayed clear Trpv4 signals in adult cortex and white matter (Fig. 1B). Hence, we demonstrated that TRPV4 is expressed in OPCs and mature oligodendrocytes. Next, to examine TRPV4 expression in astrocytes, we performed double FISH of Trpv4 and gfap (an astrocyte marker). Different from the dynamic changes of the numbers of Trpv4/PDGFR $\alpha$ double-positive cells between cerebral cortex and corpus callosum from P4 to adult, the numbers of Trpv4/gfap double-positive cells (arrowheads) between cerebral cortex and corpus callosum were not different from P4 to adult (Fig. 1C). The majority of gfap $^{+}$ astrocytes displayed clear Trpv4 signals; however, the signals were weak in some of cells. Notably, we observed many Trpv4 $/$ gafp ${ }^{+}$cells, in the adult corpus callosum (Fig. 1C), consistent with our previous reports describing TRPV4 ${ }^{+}$-astrocytes formed a specific sub-population [40, 41]. Furthermore, we confirmed the microglial TRPV4 expression by combinatorial method of 
FISH (Trpv4) and immunohistochemistry (Iba1, a microglial marker) at P4. Consistent with our previous report [21], the Iba1-positive microglial cells also express the Trpv4 in both cerebral cortex and corpus callosum (Fig. 1D). Thus, we demonstrated that TRPV4 is expressed in oligodendrocyte lineage cells in addition to the astrocytes and microglial cells in vivo.

\section{TRPV4 is functionally expressed in cultured rat OPCs}

Next, we investigated the physiological function of TRPV4 in primary cultured rat OPCs. At 2 days after replating, cells highly expressed the OPC marker PDGFR $\alpha$, but not the mature oligodendrocyte marker MBP. However, at 7 days after replating, by which time cells had been cultured in differentiation medium containing T3 and CNTF for 5 days, cells highly expressed MBP and lowly expressed PDGFR $\alpha$ (Fig. 2A). Next, we found that Trpv4 mRNA was detected by RT-PCR (Fig. 2B) and TRPV4 protein was detected by western blotting in OPCs cultured for 2 days in proliferation medium (Fig. 2C). To investigate whether TRPV4 is functionally expressed in OPCs, we measured the $\left[\mathrm{Ca}^{2+}\right]_{\mathrm{i}}$ using Fura-2 AM. Treatment with the selective TRPV4 agonist GSK1016790A increased the $\left[\mathrm{Ca}^{2+}\right]_{\mathrm{i}}$ of OPCs in a concentration-dependent manner (Fig. 2D, E). The increase in $\left[\mathrm{Ca}^{2+}\right]_{i}$ induced by GSK1016790A $(0.1 \mu \mathrm{M})$ was completely abolished by the selective TRPV4 antagonist HC067047 (10 $\mu \mathrm{M})$ (Fig. 2F). Repeated application of GSK1016790A $(0.1 \mu \mathrm{M})$ revealed that TRPV4 antagonism by HC067047 (10 $\mu \mathrm{M})$ was reversible (Fig. 2G). Whole-cell patch-clamp analysis revealed that extracellular application of GSK1016790A evoked a current response (Fig. 2H) and the current-voltage curves were outwardly rectifying (Fig. 2I), which is consistent with the results of a previous study using TRPV4 ${ }^{+}$microglia [21]. HC067047 $(30 \mu \mathrm{M})$ inhibited the GSK1016790A-induced current (Fig. 2H, I). These results indicate that TRPV4 is functionally expressed in cultured rat OPCs. 


\section{TRPV4 stimulation enhances the proliferation of OPCs via PKC}

To investigate whether TRPV4 stimulation increases the proliferation of OPCs, we analyzed cell viability and proliferation by the MTT and BrdU incorporation assays, respectively. GSK1016790A treatment increased cell viability in a concentration-dependent manner (Fig. 3A). The significant increase in cell viability induced by GSK1016790A $(0.1 \mu \mathrm{M})$ was completely abolished by co-treatment with HC067047 $(10 \mu \mathrm{M})$, whereas HC067047 alone did not significantly affect cell viability (Fig. 3B). The mean percentage of $\mathrm{BrdU}^{+}$proliferating cells was significantly higher in the group treated with GSK1016790A $(0.1 \mu \mathrm{M})$ than in the control group; however, this increase was completely suppressed by treatment with HC067047 (10 $\mu \mathrm{M})$ (Fig. 3C, D), suggesting that TRPV4 stimulation enhances OPC proliferation. We investigated the mechanism by which TRPV4 activation increases the proliferation of OPCs. To determine whether it is mediated by $\mathrm{Ca}^{2+}$ influx through TRPV4, we used the intracellular $\mathrm{Ca}^{2+}$ chelator BAPTA-AM. Treatment with BAPTA-AM $(3 \quad \mu \mathrm{M})$ significantly blocked GSK1016790A-induced OPC proliferation (Fig. 3D). Moreover, treatment with the PKC inhibitor bisindolylmaleimide II (BIM II; $0.5 \mu \mathrm{M}$ ) also significantly blocked this increase in OPC proliferation (Fig. 3E). These data suggest that TRPV4 stimulation promotes OPC proliferation via PKC.

\section{TRPV4 is not involved in the migration of OPCs}

To assess the other roles of TRPV4 in OPCs, we performed a scratch-wound assay using a monolayer of OPCs and examined the effect of GSK1016790A on their migration. OPCs migrated for 6-48 h; however, the number of cells in the scratched area did not significantly differ between control and GSK1016790A-treated cultures at any time point (Fig. 4A, B). The 
total cell viability at 24 and $48 \mathrm{~h}$ after the scratch and drug treatment was significantly increased in GSK1016790A-treated group (Fig. 4C), indicating the involvement of TRPV4 in cell proliferation. These results indicate that activation of TRPV4 increases OPC proliferation, but not migration. .

\section{TRPV4 stimulation does not affect the differentiation of OPCs}

To investigate whether TRPV4 stimulation promotes the differentiation of OPCs, we analyzed the mRNA levels of Pdgfr $\alpha$ and Mbp, which are markers of OPCs and mature oligodendrocytes, respectively, by quantitative PCR. In proliferation medium, the mRNA levels of Pdgfra and Mbp did not significantly differ between control and GSK1016790A $(0.1 \mu \mathrm{M})$-treated cells for $24 \mathrm{~h}$, although the former was significantly downregulated and the latter was significantly upregulated in differentiation medium (Fig. 5A, B). These results suggest that TRPV4 stimulation does not significantly affect OPC differentiation. Moreover, in differentiation medium, the mRNA levels of Pdgfr $\alpha$ and $M b p$ did not significantly differ between control and GSK1016790A (0.1 $\mu \mathrm{M}$ )-treated cells (Fig. 5C, D) for $72 \mathrm{~h}$, whereas there were no significant differences in the cell viability (Fig. 5E). Additionally, we evaluated the ratios of PDGFR $\alpha$ and MBP-positive cells in the presence or absence of GSK1016790A by immnostaining and found that the ratios of PDGFR $\alpha$ and MBP-positive cells did not significantly differ between GSK1016790A-treated group and control group, either in proliferation medium or differentiation medium (Fig. 5F-K). This confirms that TRPV4 stimulation does not significantly affect OPC differentiation and that TRPV4 increases the proliferation of OPCs by accelerating their proliferation, not by suppressing their differentiation. 


\section{DISCUSSION}

This study demonstrates that TRPV4 is expressed in OPCs in vivo and in cultured rat OPCs and that stimulation of TRPV4 by the selective agonist GSK1016790A induced $\mathrm{Ca}^{2+}$ responses, which was almost completely suppressed by the selective TRPV4 antagonist HC067047. TRPV4 stimulation enhances the proliferation of OPCs via $\mathrm{Ca}^{2+}$ influx and the PKC pathway, but not their migration or differentiation. These results indicate that TRPV4 increases the proliferation of OPCs, without affecting their ability to differentiate into mature oligodendrocytes.

In the proliferation assay of the present study, inhibition of TRPV4 by application of the specific TRPV4 antagonist HC067047 did not change the proliferation of OPCs. These results indicate that the functional involvement of TRPV4 in OPC proliferation is very limited in homeostatic conditions, consistent with non-histological abnormalities (normal white matter thickness) in TRPV4KO brain [38]. Meanwhile, TRPV4 activation by GSK1016790A significantly increased the proliferation of OPCs. This result suggests that TRPV4 might be activated under certain specific conditions in the brain, leading to the enhancement of proliferation. Indeed, the number of OPCs in the scratched area was higher in the GSK1016790A-treated group than in the control vehicle-treated group after $48 \mathrm{~h}$ scratching, although the difference was not significant. This may be due to the increased proliferation of GSK1016790A-treated cells responded to the pathological events.

Which endogenous molecules open TRPV4 in OPCs in the brain? Very recently, it is reported that lipopolysaccharides, the major components of the wall of gram-negative bacteria, directly activate the TRPV4 [2]. In demyelinated region, many inflammatory molecules (proteins, sugar chains or lipids) were released from myelin debris or damaged axons. Therefore, 
some of those molecules might activate TRPV4 in OPCs, and increase the proliferation of OPCs under demyelinated pathological conditions. TRPV4 is also activated by 5', 6'-epoxyeicosatrienoic acid [44], which is a signaling molecule formed in various cell types via metabolism of arachidonic acid. Arachidonic acid is upregulated in the brain in response to traumatic brain injury [47]. OPCs proliferate in response to CNS injury [28], and this may be mediated by stimulation of TRPV4 via arachidonic acid metabolites during brain inflammation. Hypo-osmolarity and mechanical stretch also are activators of TRPV4 [30]. Given that cells generate traction force during division [19] and the mechanically gated ion channel Piezo 1 can respond to such force in neural stem cells [36], TRPV4 may be open while OPCs are dividing and increase their proliferation. TRPV4 is also activated by white matter components. White matter is composed of many different components including extracellular matrix (ECM) such as laminin and collagen, and it is reported that laminin-2 induced oligodendrocytes spreading through the integrin signaling [8]. Interestingly, it is also reported that TRPV4 physically interacted with the integrin, and affected mechanical hyperalgegia [1]. Therefore, it is highly possible that the TRPV4 is significantly sensitized under endogenous ECM conditions in the white matter. Unfortunately, our physiological assay to reveal the involvement of TRPV4 was performed in vitro conditions. Hence, we might fail to mimic the intrinsic TRPV4 involvement in OPC physiological dynamics. We need specific in vivo experiments to reveal physiological impacts of the TRPV4 in OPCs as our next studies. Mild temperature $\left(>27-34^{\circ} \mathrm{C}\right)$, too, can activate TRPV4 [38]. Pharmacological blockade of TRPV4 by HC067047 alone did not affect the proliferation of $\mathrm{OPCs}$ at $37^{\circ} \mathrm{C}$ in this study, suggesting that body temperature alone is insufficient to open TRPV4 in OPCs in vitro. However, TRPV4 may be activated in OPCs by the synergistic effect of physiological temperature and other ligands such as arachidonic acid metabolites. The local brain temperature was recently reported to increase in response to local 
neuronal activity $[12,20]$, which suggests that OPCs in the vicinity of highly excited neurons are exposed to higher temperatures. Thus, TRPV4 may be particularly active in OPCs located around excited neurons and contribute to neuronal activity-induced OPC proliferation and subsequent myelination. Taken together, TRPV4 stimulation may increase proliferation of OPCs in the CNS in various physiological and pathological conditions.

Many studies have reported that $\mathrm{Ca}^{2+}$ signaling is involved in the proliferation of OPCs [7, 35]. Extracellular $\mathrm{Ca}^{2+}$ uptake through TRPC1 is important for OPC proliferation [34]. Our results indicate that $\mathrm{Ca}^{2+}$ signaling and PKC activation are involved in TRPV4-mediated OPC proliferation, consistent with the previous finding that PKC activation is related to OPC proliferation $[4,37]$. On the other hand, TRPV4 stimulation did not affect the migration or differentiation of OPCs. However, $\mathrm{Ca}^{2+}$ influx via voltage-dependent $\mathrm{Ca}^{2+}$ channels has been reported to increase OPC migration and differentiation [6, 35]. The reason for this discrepancy is unknown. However, these channels have different kinetics and capacities to transport extracellular $\mathrm{Ca}^{2+}$ into cells. They may activate distinct $\mathrm{Ca}^{2+}$ signaling pathways via different mechanisms. Further studies are required to fully understand $\mathrm{Ca}^{2+}$ signaling in OPCs.

In conclusion, our results suggest that TRPV4 is expressed in OPCs in vivo and that TRPV4 stimulation increases OPC proliferation in vitro. Therefore, TRPV4 may involve in the regulation of number of OPCs in the brain. 


\section{Figure legends}

Fig. 1 TRPV4 is expressed in OPCs in vivo. (A) Detection of Trpv4 and PDGFR $\alpha$ mRNA in P4 and adult rat cerebral cortex (CTX) and corpus callosum (CC). Both TRPV4 $4^{+} / P D G F R \alpha^{+}$ (arrowheads) and TRPV4 $/ P D G F R \alpha$ (arrows) cells were observed. (B) Detection of Trpv4 and plp mRNA in adult rat CTX and CC. Both $T R P V 4^{+} / p l p^{+}$(arrowheads) and $T R P V 4^{+} / p l p^{-}$(arrows) cells were observed. (C) Detection of Trpv4 and gfap mRNA in P4 and adult rat CTX and CC. Both $T R P V 4^{+} / g f a p^{+}$(arrowheads) and TRPV4 $/ g f a p^{-}$(arrows) cells were observed. (D) Detection of Trpv4 mRNA and Iba1 protein in P4 rat CTX and CC. TRPV4 ${ }^{+} / \mathrm{Iba}^{+}$(arrowheads) cells were observed. The dashed lines represent the border between CTX and CC. Scale bar, $20 \mu \mathrm{m}$.

Fig. 2 TRPV4 is functionally expressed in cultured rat OPCs. (A) Immunostaining of PDGFR $\alpha$ and MBP. OPCs were cultured for 2 days in proliferation medium (Day 2). To generate mature oligodendrocytes, cells were cultured for an additional 5 days in differentiation medium (Day 7). Scale bar, $50 \mu \mathrm{m}$. (B) RT-PCR of Trpv4. Cells were cultured for 2 days in proliferation medium. The marker (M) shows a 100 bp DNA ladder. (C) Western blot image of TRPV4. OPCs were cultured for 2 days in proliferation medium. (D, E) $\mathrm{Ca}^{2+}$ responses evoked by treatment with the selective TRPV4 agonist GSK1016790A at the indicated concentrations (0.01-0.1 $\mu \mathrm{M})$. (F) Changes in the $\left[\mathrm{Ca}^{2+}\right]_{\mathrm{i}}$ after administration of GSK1016790A $(0.1 \mu \mathrm{M})$ in the presence of the selective TRPV4 antagonist HC067047 $(10 \mu \mathrm{M})$. (G) Changes in the $\left[\mathrm{Ca}^{2+}\right]_{\mathrm{i}}$ after perfusion of GSK1016790A $(0.1 \mu \mathrm{M})$ with or without HC067047 $(10 \mu \mathrm{M})$. (H, I) Whole-cell current recording following treatment with GSK1016790A $(0.1 \mu \mathrm{M})$ alone or together with HC067047 (30 $\mu \mathrm{M})$. A typical trace is shown in $(\mathrm{H})$, and the current-voltage relationship is shown in (I). The hatched bars and white bars denote when GSK1016790A and HC067047 were applied, respectively. 
Fig. 3 TRPV4 stimulation enhances the proliferation of OPCs via PKC. (A, B) OPCs were treated with the TRPV4 selective agonist GSK1016790A at the indicated concentrations and with the TRPV4 selective antagonist HC067047 $(10 \mu \mathrm{M})$ for 24 h. ${ }^{*} P<0.05$, ${ }^{* * *} P<0.001$ vs. control; \#\#\# < 0.001 vs. GSK1016790A $(0.1 \mu \mathrm{M})$ alone; $n=6-8$. OPC viability was evaluated by the MTT assay. (C) Representative confocal microscopy images of BrdU incorporation by OPCs cultured in control medium (upper) and GSK1016790A (0.1 $\mu \mathrm{M})$-containing medium (lower). Proliferating OPCs and total cells were identified by BrdU and DAPI staining, respectively. Scale bar, $50 \mu \mathrm{m}$. (D, E) The percentage of $\mathrm{DAPI}^{+}$cells that were $\mathrm{BrdU}^{+}$in each experimental condition. Cells were treated with drugs together with GSK1016790A (0.1 $\mu \mathrm{M})$ for 48 h. All data are expressed as mean \pm SEM. ${ }^{*} P<0.05$ vs. control; \#P $<0.01$ vs. $\operatorname{GSK1016790A~}(0.1 \mu \mathrm{M})$ alone; $n=3$.

Fig. 4 TRPV4 stimulation does not affect the migration of OPCs. (A) OPC migration was evaluated by the scratch-wound assay. The number of cells that migrated into the scratched area was counted at the indicated time points in the control (open columns) and GSK1016790A (0.1 $\mu \mathrm{M}$; filled columns)-treated groups. $n=4$. All data are expressed as mean \pm SEM. (B) Representative images of OPCs treated with vehicle (left) or GSK1016790A (0.1 $\mu \mathrm{M}$; right) obtained at $0 \mathrm{~h}$ (upper) and $48 \mathrm{~h}$ (lower) after generation of the scratch. The black line indicates the scratch edge. Scale bar, $100 \mu \mathrm{m}$. (C) Evaluation of OPC viability after the scratch. Cell viability was evaluated by MTT assay. OPCs were treated with vehicle or GSK1016790A (0.1 $\mu \mathrm{M})$ for 24 or $48 \mathrm{~h}$ after the scratch. ${ }^{* *} P<0.01$ vs. control; $n=3$. All data are expressed as mean \pm SEM. 
Fig. 5 TRPV4 stimulation does not affect the differentiation of OPCs. (A-D) Two days after replating, cells were treated with or without GSK1016790A $(0.1 \mu \mathrm{M})$ in proliferation medium (Pro) for $24 \mathrm{~h}$ or in differentiation medium (Dif) for $72 \mathrm{~h}$. The mRNA levels of Pdgfra (A, C) and $M b p(B, D)$ were quantified by quantitative RT-PCR analysis. $n=3-4$. (E) Cell viability evaluated by MTT assay after $72 \mathrm{~h}$ treatment in proliferation medium or differentiation medium with or without GSK1016790A $(0.1 \mu \mathrm{M}) . n=4$. (F, H, I). After the drug treatment for $24 \mathrm{~h}$, cells were immunostained with anti-PDGFR $\alpha$ and MBP antibody. Panel F shows representative images of cells cultured in control proliferation medium (top), GSK1016790A (0.1 $\mu \mathrm{M}$ )-containing proliferation medium (middle) and differentiation medium (bottom). Scale bar, $50 \mu \mathrm{m}$. The quantified data of PDGFR $\alpha$ - and MBP-positive cells are shown in panel $\mathrm{H}$ and I, respectively. $n=3$. (G, J, K). After the drug treatment for $72 \mathrm{~h}$, cells were immunostained with anti-PDGFR $\alpha$ and MBP antibody. Panel G shows representative images of cells cultured in proliferation medium (top), control differentiation medium (middle) and GSK1016790A (0.1 $\mu \mathrm{M}$ )-containing differentiation medium (bottom). Scale bar, $50 \mu \mathrm{m}$. The quantified data of PDGFR $\alpha$ - and MBP-positive cells are shown in panel $\mathrm{J}$ and K, respectively. $n=3$. ${ }^{*} P<0.05$, ${ }^{* *} P<0.01,{ }^{* * *} P<0.001$ vs. control cells cultured in proliferation medium All data are expressed as mean \pm SEM. 


\section{References}

1. Alessandri-Haber N, Dina OA, Joseph EK, Reichling DB, Levine JD (2008) Interaction of transient receptor potential vanilloid 4, integrin, and SRC tyrosine kinase in mechanical hyperalgesia. J Neurosci. 28:1046-57. doi: 10.1523/JNEUROSCI.4497-07.2008

2. Alpizar YA, Boonen B, Sanchez A, Jung C, López-Requena A, Naert R, Steelant B, Luyts K, Plata C, De Vooght V, Vanoirbeek JAJ, Meseguer VM, Voets T, Alvarez JL, Hellings PW, Hoet PHM, Nemery B, Valverde MA, Talavera K (2017) TRPV4 activation triggers protective responses to bacterial lipopolysaccharides in airway epithelial cells. Nat Commun 8:1059. doi: 10.1038/s41467-017-01201-3

3. Baron W, Metz B, Bansal R, Hoekstra D, de Vries H (2000) PDGF and FGF-2 signaling in oligodendrocyte progenitor cells: regulation of proliferation and differentiation by multiple intracellular signaling pathways. Mol Cell Neurosci 15:314-329. doi: 10.1006/mcne.1999.0827

4. Bhat NR, Hauser KF, Kindy MS (1992) Cell proliferation and protooncogene induction in oligodendroglial progenitors. J Neurosci Res 32:340-349. doi: 10.1002/jnr.490320306

5. Chen LX, Ma SM, Zhang P, Fan ZC, Xiong M, Cheng GQ, Yang Y, Qiu ZL, Zhou WH, Li J (2015) Neuroprotective effects of oligodendrocyte progenitor cell transplantation in premature rat brain following hypoxic-ischemic injury. PLoS One 10:e0115997. doi: 10.1371/journal.pone.0115997

6. Cheli VT, Santiago González DA, Spreuer V, Paez PM (2015) Voltage-gated Ca2+ entry promotes oligodendrocyte progenitor cell maturation and myelination in vitro. Exp Neurol 265:69-83. doi: 10.1016/j.expneurol.2014.12.012 
7. Cheli VT, Santiago González DA, Namgyal Lama T, Spreuer V, Handley V, Murphy GG, Paez PM (2016) Conditional Deletion of the L-Type Calcium Channel Cav1.2 in Oligodendrocyte Progenitor Cells Affects Postnatal Myelination in Mice. J Neurosci 36:10853-10869. doi:10.1523/JNEUROSCI.1770-16.2016

8. Chun SJ, Rasband MN, Sidman RL, Habib AA, Vartanian T (2003) Integrin-linked kinase is required for laminin-2-induced oligodendrocyte cell spreading and CNS myelination. $\mathrm{J}$ Cell Biol.163:397-408. doi: 10.1083/jcb.200304154

9. Cui QL, Kuhlmann T, Miron VE, Leong SY, Fang J, Gris P, Kennedy TE, Almazan G, Antel J (2013) Oligodendrocyte progenitor cell susceptibility to injury in multiple sclerosis. Am J Pathol 183:516-525. doi: 10.1016/j.ajpath.2013.04.016

10. Fannon J, Tarmier W, Fulton D (2015) Neuronal activity and AMPA-type glutamate receptor activation regulates the morphological development of oligodendrocyte precursor cells. Glia 63:1021-1035. doi: 10.1002/glia.22799

11. Faulkner J, Keirstead HS (2005) Human embryonic stem cell-derived oligodendrocyte progenitors for the treatment of spinal cord injury. Transpl Immunol 15:131-142. doi: 10.1016/j.trim.2005.09.007

12. Fekete Z, Csernai M, Kocsis K, Horváth ÁC, Pongrácz A, Barthó P (2017) Simultaneous in vivo recording of local brain temperature and electrophysiological signals with a novel neural probe. J Neural Eng 14:034001. doi: 10.1088/1741-2552/aa60b1

13. Ferent J, Zimmer C, Durbec P, Ruat M, Traiffort E (2013) Sonic Hedgehog signaling is a positive oligodendrocyte regulator during demyelination. J Neurosci 33:1759-1772. doi:10.1523/JNEUROSCI.3334-12.2013

14. Fornari E, Maeder P, Meuli R, Ghika J, Knyazeva MG (2012) Demyelination of superficial white matter in early Alzheimer's disease: a magnetization transfer imaging 
study. a magnetization transfer imaging study. Neurobiol Aging 428.e7-19. doi: 10.1016/j.neurobiolaging.2010.11.014

15. Frohman EM, Racke MK, Raine CS (2006) Multiple sclerosis--the plaque and its pathogenesis. N Engl J Med 354:942-955. doi: 10.1056/NEJMra052130

16. Hamilton N, Vayro S, Wigley R, Butt AM (2010) Axons and astrocytes release ATP and glutamate to evoke calcium signals in NG2-glia. Glia 58:66-79. doi: 10.1002/glia.20902

17. Hamilton NB, Kolodziejczyk K, Kougioumtzidou E, Attwell D (2016) Proton-gated $\mathrm{Ca}(2+)$-permeable TRP channels damage myelin in conditions mimicking ischaemia. Nature 529: 523-527. doi: 10.1038/nature16519

18. Hoffmann A, Grimm C, Kraft R, Goldbaum O, Wrede A, Nolte C, Hanisch UK, Richter-Landsberg C, Brück W, Kettenmann H, Harteneck C (2010) TRPM3 is expressed in sphingosine-responsive myelinating oligodendrocytes. J Neurochem 114:654-665. doi: 10.1111/j.1471-4159.2010.06644.x

19. Jahan MGS, Yumura S (2017) Traction force and its regulation during cytokinesis in Dictyostelium cells. Eur J Cell Biol 96:515-528. doi: 10.1016/j.ejcb.2017.06.004

20. Kiyatkin EA, Brown PL, Wise RA (2002) Brain temperature fluctuation: a reflection of functional neural activation. Eur J Neurosci 16:164-8.

21. Konno M, Shirakawa H, Iida S, Sakimoto S, Matsutani I, Miyake T, Kageyama K, Nakagawa T, Shibasaki K, Kaneko S (2012) Stimulation of transient receptor potential vanilloid 4 channel suppresses abnormal activation of microglia induced by lipopolysaccharide. Glia 60:761-770. doi: 10.1002/glia.22306

22. Kuroda M, Muramatsu R, Maedera N, Koyama Y, Hamaguchi M, Fujimura H, Yoshida M, Konishi M, Itoh N, Mochizuki H, Yamashita T (2017) Peripherally derived FGF21 promotes remyelination in the central nervous system. J Clin Invest 127:3496-3509. doi: 


\subsection{2/JCI94337}

23. Lee Y, Morrison BM, Li Y, Lengacher S, Farah MH, Hoffman PN, Liu Y, Tsingalia A, Jin L, Zhang PW, Pellerin L, Magistretti PJ, Rothstein JD (2012) Oligodendroglia metabolically support axons and contribute to neurodegeneration. Nature 487:443-448. doi: 10.1038/nature11314

24. Li C, Xiao L, Liu X, Yang W, Shen W, Hu C, Yang G, He C (2013) A functional role of NMDA receptor in regulating the differentiation of oligodendrocyte precursor cells and remyelination. Glia 61:732-749. doi: 10.1002/glia.22469

25. Lundgaard I, Luzhynskaya A, Stockley JH, Wang Z, Evans KA, Swire M, Volbracht K, Gautier HO, Franklin RJ, Charles Ffrench-Constant, Attwell D, Káradóttir RT (2013) Neuregulin and BDNF induce a switch to NMDA receptor-dependent myelination by oligodendrocytes. PLoS Biol 11:e1001743. doi: 10.1371/journal.pbio.1001743

26. Ma J, Matsumoto M, Tanaka KF, Takebayashi H, Ikenaka K (2006) An animal model for late onset chronic demyelination disease caused by failed terminal differentiation of oligodendrocytes. Neuron Glia Biol 2:81-91. doi: 10.1017/S1740925X06000056

27. Ma J, Tanaka KF, Yamada G, Ikenaka K (2007) Induced expression of cathepsins and cystatin C in a murine model of demyelination. Neurochem Res 32:311-320. doi: 10.1007/s11064-006-9183-y

28. McTigue DM, Wei P, Stokes BT (2001) Proliferation of NG2-positive cells and altered oligodendrocyte numbers in the contused rat spinal cord. J Neurosci 21:3392-3400.

29. Miyake T, Nakamura S, Zhao M, So K, Inoue K, Numata T, Takahashi N, Shirakawa H, Mori Y, Nakagawa T, Kaneko S (2016) Cold sensitivity of TRPA1 is unveiled by the prolyl hydroxylation blockade-induced sensitization to ROS. Nat Commun 7:12840. doi: 10.1038/ncomms12840. 
30. Mochizuki T, Sokabe T, Araki I, Fujishita K, Shibasaki K, Uchida K, Naruse K, Koizumi S, Takeda M, Tominaga M (2009) The TRPV4 cation channel mediates stretch-evoked $\mathrm{Ca}^{2+}$ influx and ATP release in primary urothelial cell cultures. J Biol Chem 287:21257-21264. doi: 10.1074/jbc.M109.020206

31. Najjar S, Pearlman DM (2016) Neuroinflammation and white matter pathology in schizophrenia: systematic review. Schizophr Res 161:102-112. doi: 10.1016/j.schres.2014.04.041

32. Ortega F, Gascón S, Masserdotti G, Deshpande A, Simon C, Fischer J, Dimou L, Chichung Lie D, Schroeder T, Berninger B (2013) Oligodendrogliogenic and neurogenic adult subependymal zone neural stem cells constitute distinct lineages and exhibit differential responsiveness to Wnt signalling. Nat Cell Biol 15:602-613. doi: $10.1038 /$ ncb2736

33. Paez PM, Fulton DJ, Spreur V, Handley V, Campagnoni AT (2010) Multiple kinase pathways regulate voltage-dependent $\mathrm{Ca} 2+$ influx and migration in oligodendrocyte precursor cells. J Neurosci 30:6422-33. doi: 10.1523/JNEUROSCI.5086-09.2010

34. Paez PM, Fulton D, Spreuer V, Handley V, Campagnoni AT (2011) Modulation of canonical transient receptor potential channel 1 in the proliferation of oligodendrocyte precursor cells by the golli products of the myelin basic protein gene. J Neurosci 31:3625-3637. doi: 10.1523/JNEUROSCI.4424-10.2011

35. Paez PM, Cheli VT, Ghiani CA, Spreuer V, Handley VW, Campagnoni AT (2012) Golli myelin basic proteins stimulate oligodendrocyte progenitor cell proliferation and differentiation in remyelinating adult mouse brain. Glia 60:1078-1093. doi: 10.1002/glia.22336

36. Pathak MM, Nourse JL, Tran T, Hwe J, Arulmoli J, Le DT, Bernardis E, Flanagan LA, 
Tombola F (2014) Stretch-activated ion channel Piezo1 directs lineage choice in human neural stem cells. Proc Natl Acad Sci U S A 111:16148-53. doi: 10.1073/pnas.1409802111

37. Radhakrishna M, Almazan G (1994) Protein kinases mediate basic fibroblast growth factor's stimulation of proliferation and c-fos induction in oligodendrocyte progenitors. Brain Res Mol Brain Res 24:118-128.

38. Shibasaki K, Suzuki M, Mizuno A, Tominaga M (2007) Effects of body temperature on neural activity in the hippocampus: regulation of resting membrane potentials by transient receptor potential vanilloid 4. J Neurosci 27:1566-1575. doi: 10.1523/JNEUROSCI.4284-06.2007

39. Shibasaki K, Takebayashi H, Ikenaka K, Feng L, Gan L (2007) Expression of the basic helix-loop-factor Olig2 in the developing retina: Olig2 as a new marker for retinal progenitors and late-born cells. Gene Expr Patterns 7:57-65. doi: 10.1016/j.modgep.2006.05.008

40. Shibasaki K, Ikenaka K, Tamalu F, Tominaga M, Ishizaki Y (2014) A novel subtype of astrocytes expressing TRPV4 (transient receptor potential vanilloid 4) regulates neuronal excitability via release of gliotransmitters. The Journal of biological chemistry 289:14470-14480. doi:10.1074/jbc.M114.557132

41. Shibasaki K (2016) TRPV4 ion channel as important cell sensors. Journal of Anesthesia 30:1014-1019. doi: 10.1007/s00540-016-2225-y

42. Shirakawa H, Katsumoto R, Iida S, Miyake T, Higuchi T, Nagashima T, Nagayasu K, Nakagawa T, Kaneko S (2017) Sphingosine-1-phosphate induces $\mathrm{Ca}^{2+}$ signaling and CXCL1 release via TRPC6 channel in astrocytes. Glia 65:1005-1016. doi: 10.1002/glia.23141 
43. Sypecka J, Sarnowska A (2014) The neuroprotective effect exerted by oligodendroglial progenitors on ischemically impaired hippocampal cells. Mol Neurobiol 49:685-701. doi: 10.1007/s12035-013-8549-9

44. Watanabe H, Vriens J, Prenen J, Droogmans G, Voets T, Nilius B (2003) Anandamide and arachidonic acid use epoxyeicosatrienoic acids to activate TRPV4 channels. Nature 424:434-438. doi: 10.1038/nature01807

45. Waxman SG, Bennett MV (1972) Relative conduction velocities of small myelinated and non-myelinated fibres in the central nervous system. Nat New Biol 85:217-219.

46. Wu LJ, Sweet TB, Clapham DE (2010) International Union of Basic and Clinical Pharmacology. LXXVI. Current progress in the mammalian TRP ion channel family. Pharmacol Rev 62:381-404. doi: 10.1124/pr.110.002725

47. Zheng F, Xia ZA, Zeng YF, Luo JK, Sun P, Cui HJ, Wang Y, Tang T, Zhou YT (2017) Plasma metabolomics profiles in rats with acute traumatic brain injury. PLoS One 12:e0182025. doi: 10.1371/journal.pone.0182025 
Fig. 1
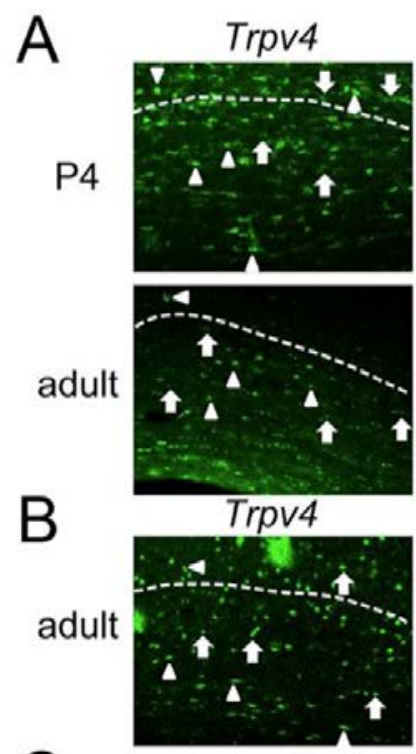

C

P4

D

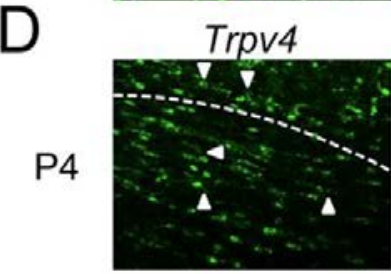

PDGFR $\alpha$

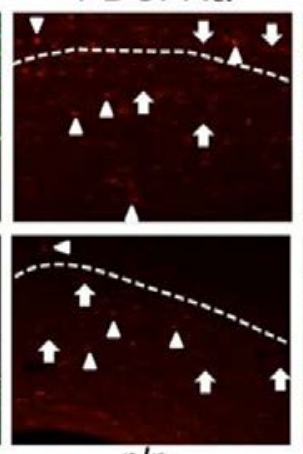

$p / p$

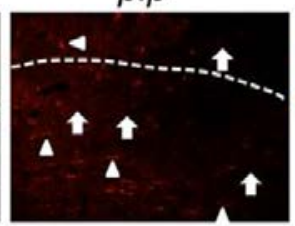

gfap

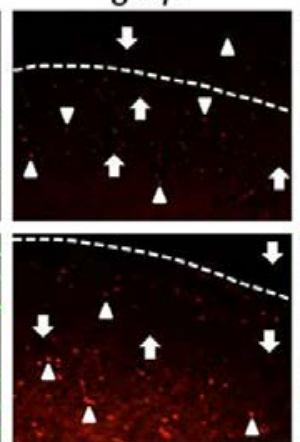

Iba1

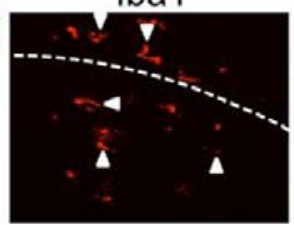

Merged

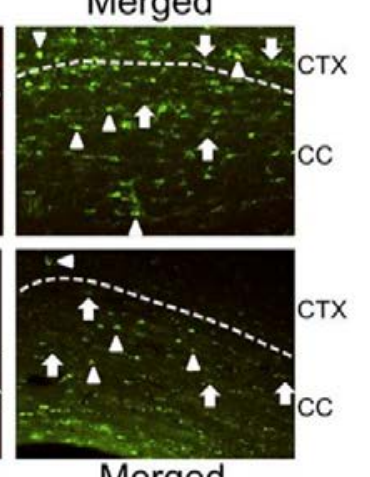

Merged

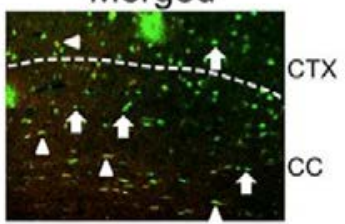

Merged

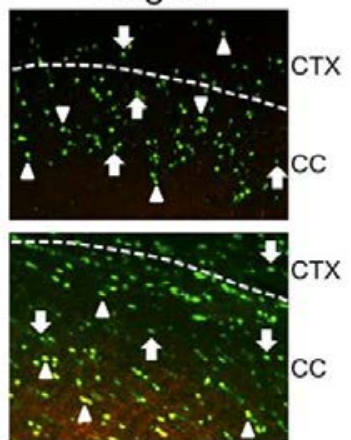

Merged

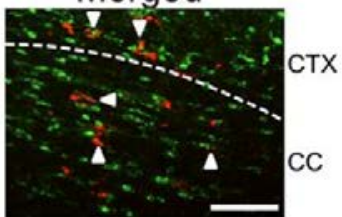

A

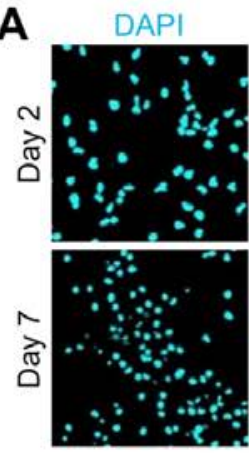

B
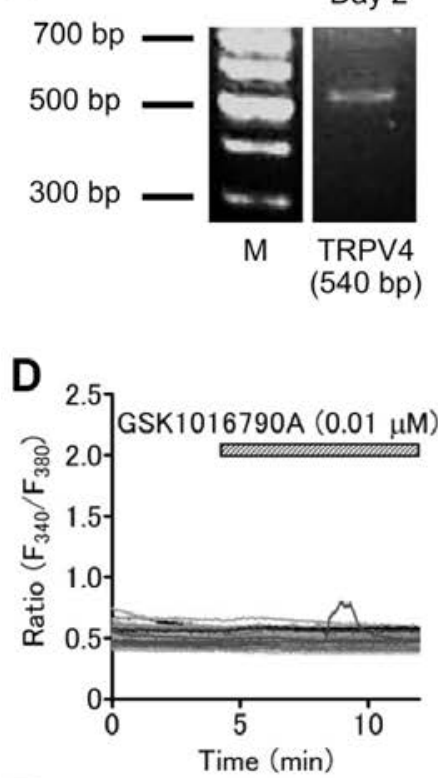

F

H

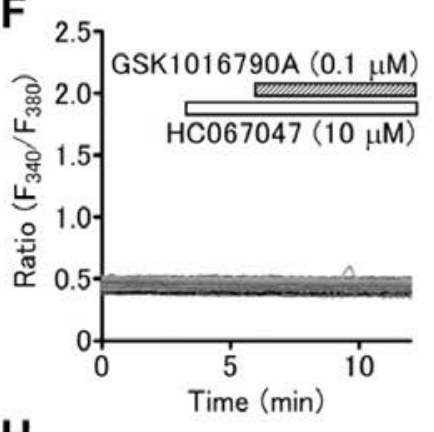

口GSK1016790A (0.1 $\mu \mathrm{M})$

마C067047 (30 $\mu \mathrm{M})$

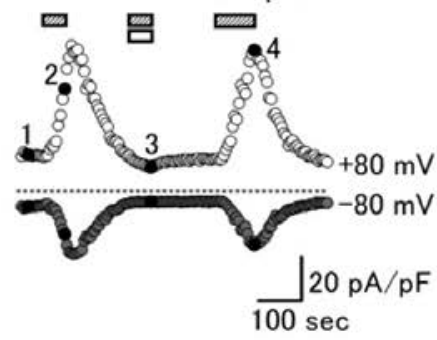

Fig. 2

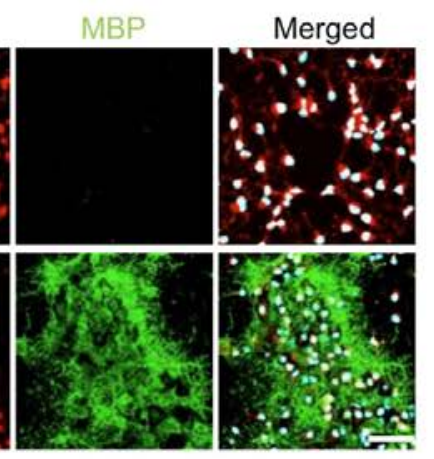

C

Day 2

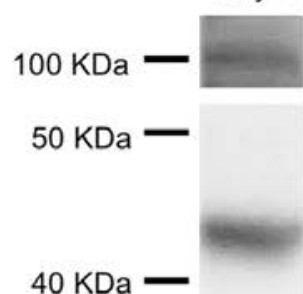

TRPV4

Actin

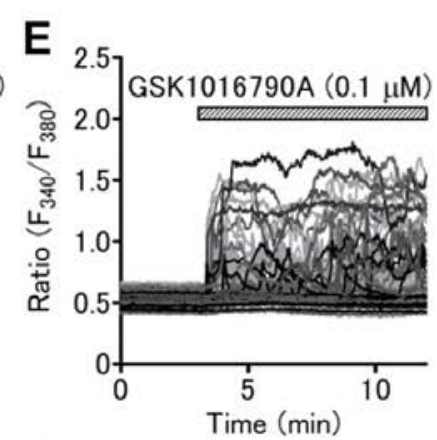

G 2.5 gGSK1016790A (0.1 $\mu \mathrm{M})$
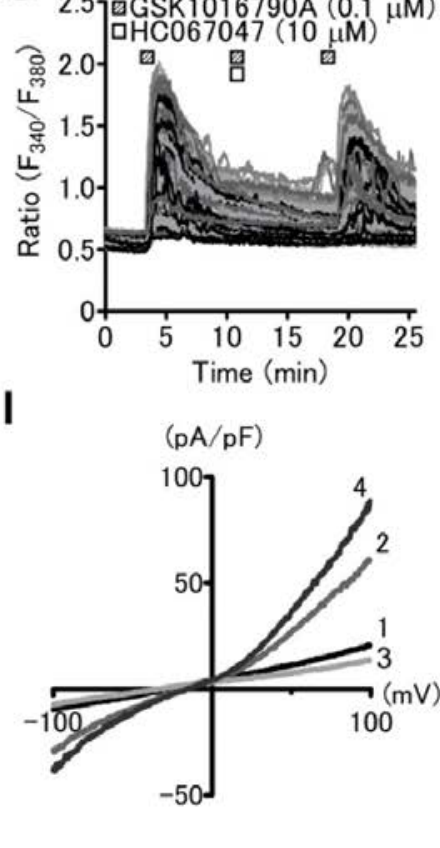
Fig. 3

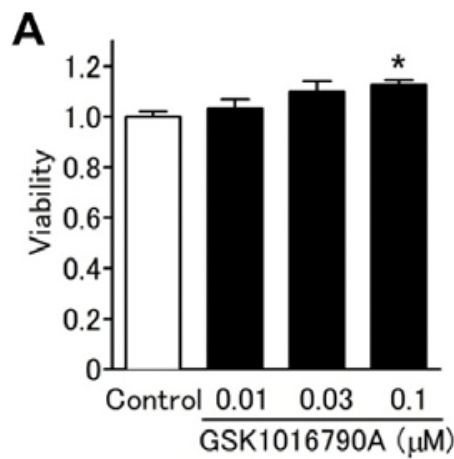

C

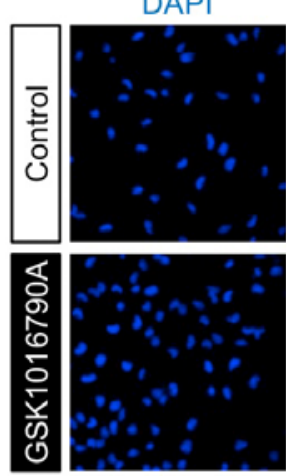

B

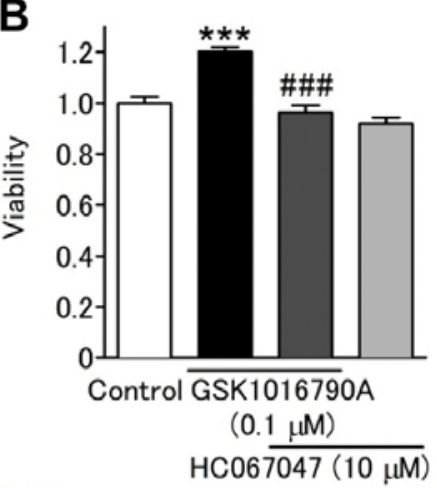

BrdU

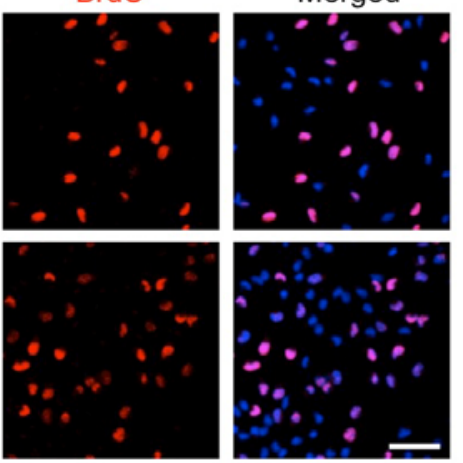

D

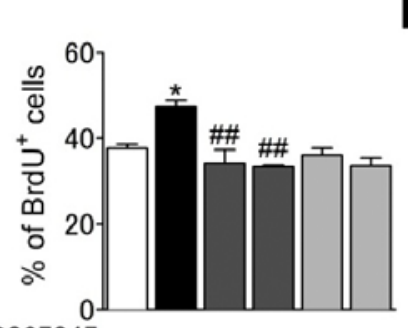

$\mathrm{HC} 06704$

$(10 \mu \mathrm{M})$

BAPTA-AM _ _ +++

$(3 \mu \mathrm{M})$

GSK $\overline{1016790 A}(0.1 \mu \mathrm{M})$
E

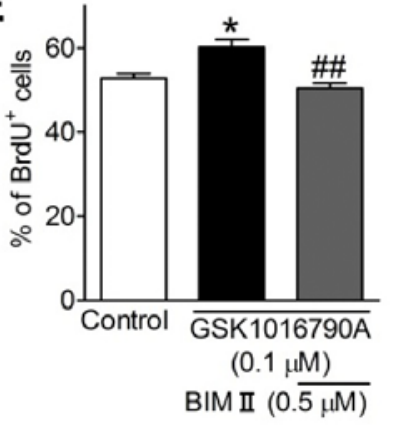

A

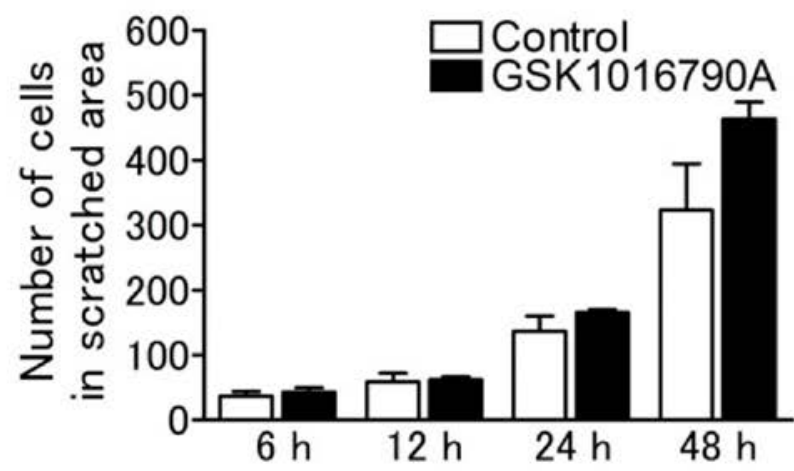

B

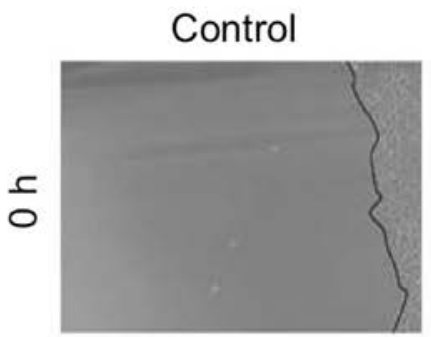

ᄃ

C

$24 \mathrm{~h}$

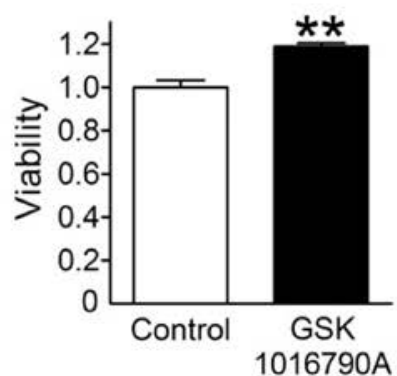

GSK1016790A

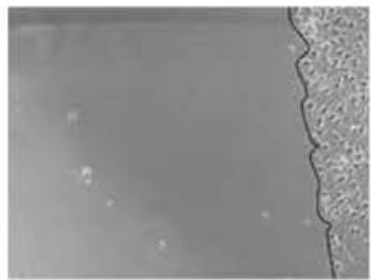

$48 \mathrm{~h}$

Fig. 4
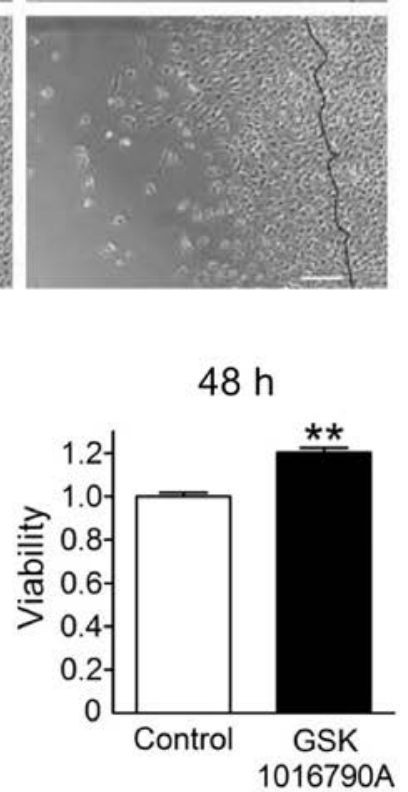
